\title{
Folic acid conjugation improves the bioavailability and chemosensitizing efficacy of curcumin-encapsulated PLGA-PEG nanoparticles towards paclitaxel chemotherapy
}

\author{
Arun Kumar T. Thulasidasan ${ }^{1,3, *}$, Archana P. Retnakumari ${ }^{1, *}$, Mohan Shankar ${ }^{1,4}$, \\ Vinod Vijayakurup ${ }^{1}$, Shabna Anwar ${ }^{1,3}$, Sanu Thankachan ${ }^{1}$, Kavya S. Pillai ${ }^{1}$, Jisha J. \\ Pillai $^{2}$, C. Devika Nandan ${ }^{2}$, Vijai V. Alex ${ }^{1}$, Teena Jacob Chirayil ${ }^{2,3}$, Sankar Sundaram ${ }^{5}$, \\ Gopalakrishnapillai Sankaramangalam Vinod Kumar ${ }^{2}$ and Ruby John Anto ${ }^{1}$ \\ ${ }^{1}$ Division of Cancer Research, Rajiv Gandhi Centre for Biotechnology, Thiruvananthapuram, Kerala, India \\ ${ }^{2}$ Division of Chemical Biology-Nano Drug Delivery Systems, Rajiv Gandhi Centre for Biotechnology, Thiruvananthapuram, \\ Kerala, India \\ ${ }^{3}$ Research Scholar, University of Kerala, Thiruvananthapuram, Kerala, India \\ ${ }^{4}$ Research Scholar, Manipal University, Manipal, Karnataka, India \\ ${ }^{5}$ Department of Pathology, Government Medical College, Kottayam, Kerala, India \\ *These authors contributed equally to this work \\ Correspondence to: Ruby John Anto, email: rjanto@rgcb.res.in
}

Keywords: curcumin; folic acid conjugation; PLGA nanoparticles; bioavailability; chemosensitization

Received: May 31, $2017 \quad$ Accepted: October 25, $2017 \quad$ Published: November 10, 2017

Copyright: Thulasidasan et al. This is an open-access article distributed under the terms of the Creative Commons Attribution License 3.0 (CC BY 3.0), which permits unrestricted use, distribution, and reproduction in any medium, provided the original author and source are credited.

\section{ABSTRACT}

Nanoencapsulation has emerged as a novel strategy to enhance the pharmacokinetic and therapeutic potential of conventional drugs. Recent studies from our lab have established the efficacy of curcumin in sensitizing cervical cancer cells and breast cancer cells towards paclitaxel and 5-FU chemotherapy respectively. Factors that hinder the clinical use of curcumin as a sensitizer or therapeutic agent include its poor bioavailability and retention time. Earlier reports of improvement in bioavailability and retention of drugs upon nanoencapsulation have motivated us in developing various nanoformulations of curcumin, which were found to exhibit significant enhancement in bioavailability and retention time as assessed by our previous in vitro studies. Among the various formulations tested, curcumin-entrapped in PLGA-PEG nanoparticles conjugated to folic acid (PPF-curcumin) displayed maximum cell death. In the present study, we have demonstrated the efficacy of this formulation in augmenting the bioavailability and retention time of curcumin, in vivo, in Swiss albino mice. Further, the acute and chronic toxicity studies proved that the formulation is pharmacologically safe. We have also evaluated its potential in chemosensitizing cervical cancer cells to paclitaxel and have verified the results using cervical cancer xenograft model in NOD-SCID mice. Folic acid conjugation significantly enhanced the efficacy of curcumin in down-regulating various survival signals induced by paclitaxel in cervical cancer cells and have considerably improved its potential in inhibiting the tumor growth of cervical cancer xenografts. The non-toxic nature coupled with improved chemosensitization potential makes PPF-curcumin a promising candidate formulation for clinical trials. 


\section{INTRODUCTION}

Paclitaxel is one of the most potent naturally derived chemotherapeutic agents discovered till date [1]. It is an anti-mitotic agent that promotes apoptosis in cancer cells by stabilizing microtubules, and has been widely used for treating several cancers $[2,3]$. However, its efficacy as an anticancer agent keeps declining as cells become resistant to the normally administered dose, thus creating a need for exposure to higher doses of the drug leading to more deleterious side effects [4]. Pre-clinical and clinical studies have identified multitude of resistance mechanisms such as, altered metabolism of the drug [5], over-expression of the drug transporter p-glycoprotein [6], mutations in the target molecule $\beta$-tubulin [7], alterations in the apoptotic signaling mechanisms [5, 7] etc. Owing to the hydrophobic nature of paclitaxel, it is administered in Cremophor EL solvent which has also been reported to induce toxicity. Although, Nab paclitaxel (paclitaxel bound to albumin nanoparticles) could overcome most of the limitations of conventional paclitaxel [8], it is highly expensive and cannot be afforded by common people. Hence, a more feasible and cost-effective option would be to sensitize the cancer cells towards paclitaxel chemotherapy using nontoxic chemosensitizers which when used in combination, bring down the effective dose of paclitaxel to be used. Previous investigations from our lab have illustrated the potential of curcumin, a polyphenol derived from Curcuma longa, in chemosensitizing cervical cancer cells to paclitaxel [9-11] and breast cancer cells to 5-flurouracil [12]. Curcumin has been found to be effective in suppressing paclitaxel-induced NF- $\mathrm{kB}$ pathway in breast cancer cells and lung cancer cells [13]. However, the major drawbacks in using curcumin as a chemosensitizer in vivo were its poor aqueous solubility leading to its fast clearance and poor bio-availability at the target site [14]. Encapsulation of curcumin in nanoparticles has been proved as a feasible strategy to improve the circulation and absorption of highly hydrophobic drugs [15]. Co-administration of paclitaxel and curcumin as nanoemulsions has been shown to overcome multidrug resistance in tumor cells by Ganta S et al. [16]. Our in vitro studies have successfully demonstrated that, encapsulation of curcumin in PLGA nanoparticles conjugated with folic acid could increase the therapeutic potential of curcumin $[17,18]$. In the current study, we have carried out extensive in vitro and in vivo studies to evaluate the chemosensitizing efficacy of PPF-curcumin towards paclitaxel chemotherapy. We could successfully demonstrate that the encapsulation of curcumin in PLGA-PEG nanoparticles and further conjugation with folic acid enhanced the bioavailability and tissue retention of curcumin in vivo compared to liposomal curcumin. We have reported earlier the in vivo synergistic efficacy of paclitaxel and curcumin in NODSCID mice [11], wherein the route of administration for toxicity and tumor reduction studies were intraperitoneal. Since the present study aimed to evaluate whether folic acid conjugation can improve the tissue retention and bioavailability of curcumin encapsulated PLGA-PEG nanoparticles than liposomal curcumin (as used in the previous study), the same route of administration was used for both tumor reduction and safety studies. Our in vivo studies could successfully validate the synergistic efficacy of PPF-curcumin in paclitaxel chemotherapy and the results indicated that PPF-curcumin exhibited a superior efficacy when compared with that of liposome curcumin. Molecular level analyses have shown that PPFcurcumin is much superior in down-regulating paclitaxelinduced up-regulation of survival, proliferative and prometastatic signals. We strongly believe that the current study, illustrating the efficacy of PPF-curcumin might be a therapeutically efficient strategy for sensitizing cancer cells towards paclitaxel, which could further enhance the therapeutic outcome of paclitaxel chemotherapy.

\section{RESULTS}

\section{Encapsulation of curcumin in folic acid conjugated PLGA-PEG nanoparticles significantly improves its efficacy in chemosensitizing HeLa cells}

Our earlier studies have already established that curcumin could be used as an effective chemosensitizer in paclitaxel chemotherapy [9-11]. Curcumin encapsulated in nanoparticles prepared from PLGA-PEG block copolymer and conjugated to the tumor-targeting ligand folic acid showed significant chemosensitization potential towards paclitaxel compared to free curcumin [19]. These nanoparticles abbreviated as PPF-curcumin which showed a typical size of 100-200 nm in TEM (Supplementary Figure 1) exhibited a sustained release of curcumin in vitro. We have also observed that PPF-curcumin exhibits enhanced therapeutic efficacy in vitro, compared to either PLGAcurcumin or free curcumin [19], probably due to uptake of the cucrumin via folate receptors (FOLR1) reported to be over-expressed in almost all cancer types [20].

In the current study, we compared the synergistic cytotoxicity of free curcumin and PPF-curcumin in combination with paclitaxel, using MTT assay. Figure 1A showed that PPF-curcumin exerts enhanced chemosensitization potential towards paclitaxelinduced cytotoxicity compared to free curcumin. The combination index indicating synergistic cytotoxic effect of free curcumin and PPF-curcumin in combination with paclitaxel was determined using Compusyn software. The results are incorporated in Supplementary Table 1. The combination of $5 \mathrm{nM}$ paclitaxel and $5 \mu \mathrm{M}$ curcumin induced a synergistic cytotoxicity with a combination index CI of 0.685 , whereas the combination of $5 \mathrm{nM}$ paclitaxel and $5 \mu \mathrm{M}$ PPF-curcumin induced a synergistic 
cytotoxicity with a combination index CI of 0.315 . The chromatin condensation assay showed in Figure 1B indicates significant enhancement in apoptosis as indicated by the extensive condensation of chromatin in PPFcurcumin+paclitaxel treated cells compared to that of free curcumin+paclitaxel. The number of condensed nuclei is counted and represented as a graph. The combination of PPF-curcumin and paclitaxel also caused drastic inhibition in the clonogenic potential of HeLa cells compared to free curcumin as shown in Figure 1C. For improved clarity of the clones, 10x images of the individual wells are shown in the lower panel of Figure 1C. Even though both free curcumin and PPF-curcumin augmented paclitaxelinduced cleavage of caspase- 9 and caspase- 3 , the effect induced by PPF-curcumin was superior to that of free curcumin as indicated by Figure 1D. To make sure that the combination of PPF-curcumin and paclitaxel is not inducing synergistic cytotoxicity towards normal cells, we evaluated their cytotoxicity in the non-tumorigenic immortalized cell line, $\mathrm{HaCaT}$, which has successfully been used earlier as a suitable control by other groups [21]. In contrast to the results obtained in HeLa, neither free curcumin nor PPF-curcumin induced synergistic cytotoxicity in $\mathrm{HaCaT}$ cells in combination with paclitaxel (Supplementary Figure 2), corroborating that curcumin or PPF-curcumin does not sensitize normal cells towards paclitaxel, attesting our previous studies using normal cervical cells $[9,11]$.
A

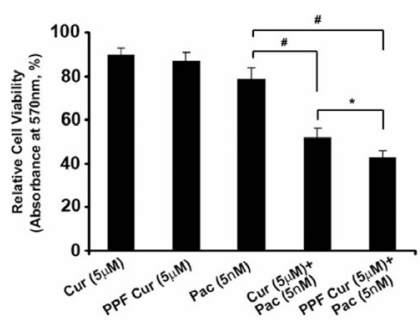

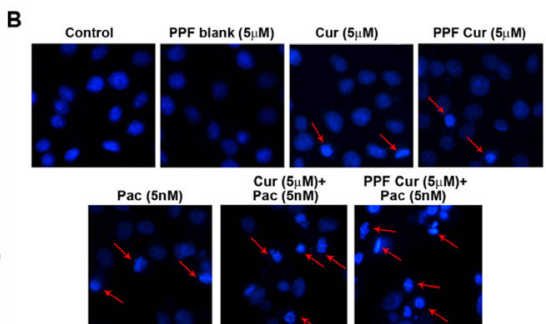

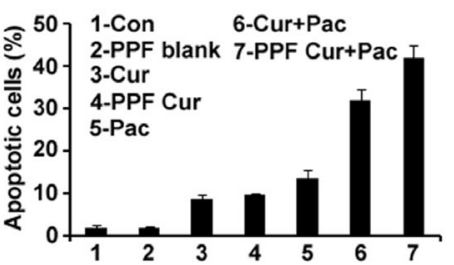

C

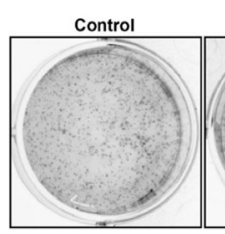

PPF Blank (5 $\mu \mathrm{M})$
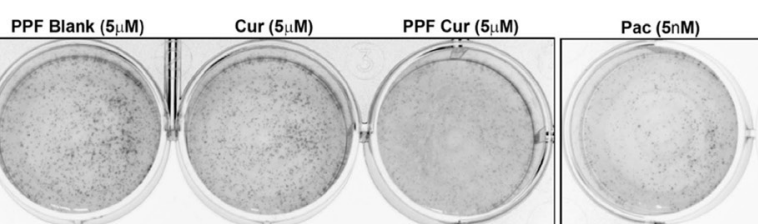

Cur (5uM)+
Pac (5nM)

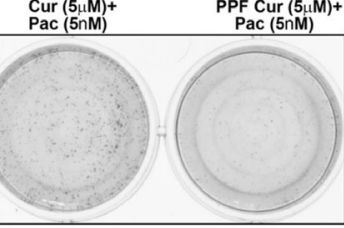

Control

PPF Blank (5 $\mu \mathrm{M})$

$\operatorname{Cur}(5 \mu \mathrm{M})$

Pac (5nM)

Cur $(5 \mu M)+$
Pac $(5 n M)$

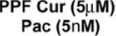

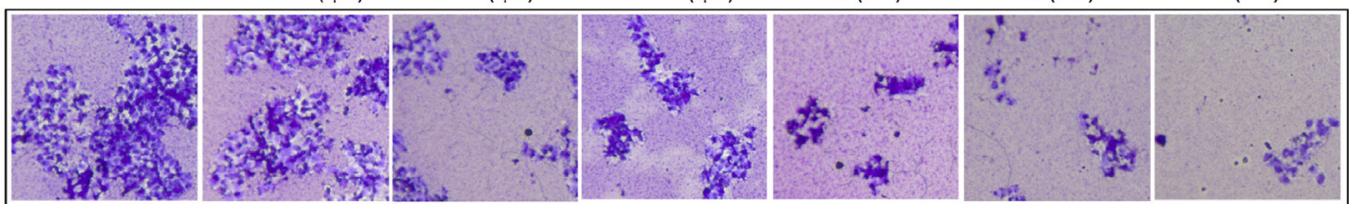

D
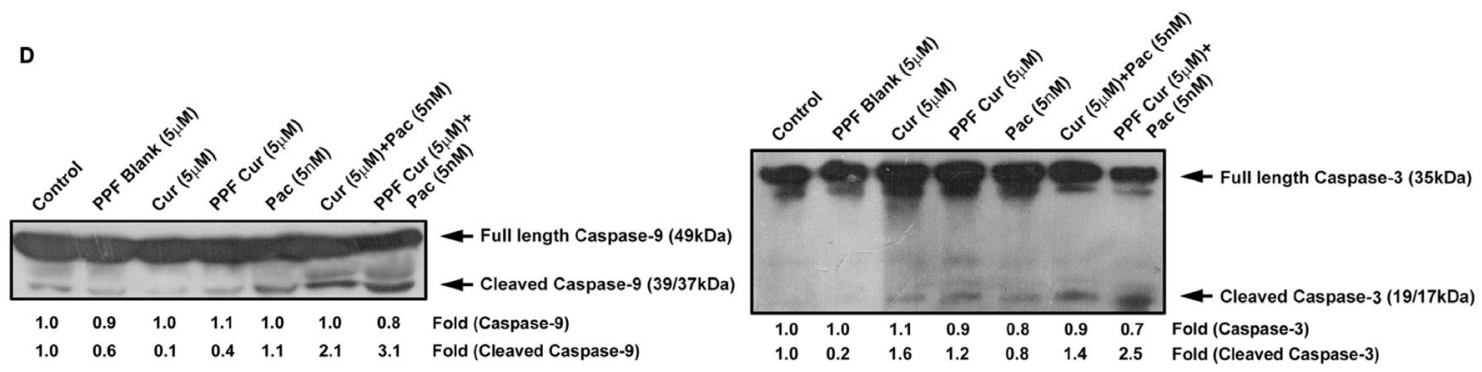

Figure 1: PPF-curcumin induces significant chemosensitization towards paclitaxel. (A) PPF-curcumin is more efficient in augmenting paclitaxel-induced cytotoxicity in HeLa cells, compared to free curcumin. HeLa cells were treated with free curcumin/PPFcurcumin either alone or in combination with paclitaxel for $72 \mathrm{~h}$ after pre-treating with curcumin/PPF-curcumin and cell viability assay was performed using MTT. (B) Paclitaxel-induced chromatin condensation is enhanced more efficiently by PPF-curcumin compared to free curcumin. HeLa cells were treated with free curcumin/PPF curcumin either alone or in combination with paclitaxel for $24 \mathrm{~h}$ after pretreating with curcumin/PPF-curcumin. Later, cells were stained with DAPI and images were captured using a fluorescent microscope. The number of condensed nuclei is counted and represented as a graph. (C) PPF-curcumin inhibits clonogenicity of HeLa cells. Cells were treated with different formulations of curcumin $(5 \mu \mathrm{M})$ either alone or in combination with paclitaxel for $72 \mathrm{~h}$ and clonogenic assay was conducted. The lower panel shows the images of individual wells captured at 10X magnification. (D) Paclitaxel-induced caspase cleavage is enhanced by PPF-curcumin more efficiently than free curcumin. HeLa cells were treated with free curcumin or PPF-curcumin either alone or in combination with paclitaxel for $24 \mathrm{~h}$ after pre-treating with curcumin/PPF-curcumin, subjected to Western blotting followed by detection by ECL. 


\section{PPF-curcumin is more efficient in down- regulating paclitaxel-induced activation of NF-kB, Akt and MAPK pathways compared to free curcumin}

The activation of NF- $\mathrm{kB}$, Akt and Mitogen Activated Protein Kinases (MAPK) are common events responsible for the survival, proliferation and drug resistance of tumor cells [22-25]. Abrogation of these pathways using small interfering RNA (siRNA) or inhibitors have been shown to cause reduction in proliferation of cancer cells as well as tumor reduction in vivo either alone or in combination with other chemotherapeutic drugs [26-29]. Prolonged exposure of chemotherapeutic drugs including paclitaxel have been shown to activate these survival signals, which in turn make the cancer cells chemo-resistant, necessitating higher doses of the drugs to elicit a desired therapeutic effect [30]. Previous in vitro and in vivo studies from our group have demonstrated the efficacy of curcumin in successfully bringing down paclitaxel-induced activation of survival pathways in cervical cancer [11]. We questioned whether encapsulation of curcumin in PPF nanoparticles can enhance its ability in down-regulating paclitaxel-induced survival signals. Figure 2A clearly indicates that, PPF-curcumin is much more efficient in down-regulating paclitaxel-induced phosphorylation of Akt compared to free curcumin. Evaluation of the DNA binding of NF- $\kappa \mathrm{B}$ by electrophoretic mobility shift assay (EMSA) as shown in Figure 2B also demonstrated that PPF-curcumin is more successful than free curcumin. Paclitaxel-induced NF- $\kappa B$ activation leads to its nuclear translocation ensuing induction of target genes such as cyclin D1, Bcl-2 and Cox-2, all of which can in turn contribute to chemoresistance. In concordance with the EMSA results, PPF-curcumin displayed better efficacy in down-regulating paclitaxel-induced up-regulation of $\mathrm{NF}-\kappa \mathrm{B}$ target genes such as Cyclin D1, Cox-2, Bcl-2, XIAP, c-IAP and survivin than free curcumin as shown in Figure 2C and 2D. Our earlier studies have revealed the regulatory role of curcumin in modulating paclitaxelinduced activation of MAPK pathway [10,11]. Figure 2E and $2 \mathrm{~F}$ clearly indicate that, PPF encapsulation drastically enhances the potential of curcumin in regulating the MAPKs, ERK1/2, JNK and p-38 and their downstream transcription factor, AP-1. Taken together the in vitro results clearly demonstrate that, PPF encapsulation significantly enhances the chemosensitizing efficacy of curcumin towards paclitaxel in HeLa cells.

\section{PPF-curcumin is pharmacologically safe in healthy Swiss albino mice}

Our next attempt was to validate the pharmacological safety of the formulation. We conducted both acute and chronic toxicity studies in healthy Swiss albino mice using both liposomal curcumin and PPF-curcumin or their blank carriers. SCID mice are immuno-compromised animals, and are usually used to grow human xenografts. As far as the toxicological evaluation of the drug is concerned, it should be given to a normal healthy animal which has all the potential to defend the toxicological parameters of the drug as the final end users of all these drugs are human beings, and hence we conducted the toxicological evaluation in healthy Swiss albino mice. We focused on the liver histopathological analysis and liver function parameters, since nanoparticles have been reported to cause hepatotoxicity [31]. Biochemical analysis of serum samples and histopathological evaluation of liver sections were performed in animals injected with either liposomal curcumin/PPF-curcumin or their blank carriers in both acute (7 days) and chronic toxicity studies (2 months). Histopathological verification of liver tissue sections as shown in Figure 3A and 3B revealed no significant morphological changes or pathological conditions at the dosage administered. The liver tissue sections did not show any sign of fatty or irreversible liver damage. Liver function enzymes also did not show any drastic fluctuations from their normal range indicating that either PPF-curcumin or liposome curcumin does not cause any significant hepatotoxicity as indicated in Figure 3C and 3D. A similar study was also conducted in the case of paclitaxel and its combination with liposomal curcumin or PPF-curcumin, which showed that in the combination also, there were no significant histopathological manifestations as assessed by acute and chronic toxicity studies (Supplementary Figure 3A and 3B, respectively) or wide variations in liver function parameters (Supplementary Figure $3 \mathrm{C}$ and $3 \mathrm{D}$, respectively).

\section{Nanoencapsulation and folic acid conjugation enhances curcumin's chemosensitization potential towards paclitaxel causing significant reduction of tumor growth in vivo with reduction in NF- $\kappa \mathrm{B}$ and $\mathrm{AP}-1$ nuclear translocation}

To assess the in vivo chemosensitization efficacy of PPF-curcumin towards paclitaxel, human cervical cancer xenografts of NOD-SCID mice were employed. Previous studies from our lab have shown that curcumin enhances the anti-tumor activity of paclitaxel against human cervical cancer xenograft model in NOD-SCID mice, where we used liposomal formulation [11]. Though significant tumor reduction was observed both in multistage carcinogenesis model and NOD-SCID xenograft models of cervical cancer, we could not achieve in vivo curcumin retention for a longer duration. The dose of paclitaxel and curcumin were used according to our earlier studies [11]. Representative images of mice from different treatment groups are shown in Figure 4A. It was interesting to note that, though curcumin or PPF- 
A

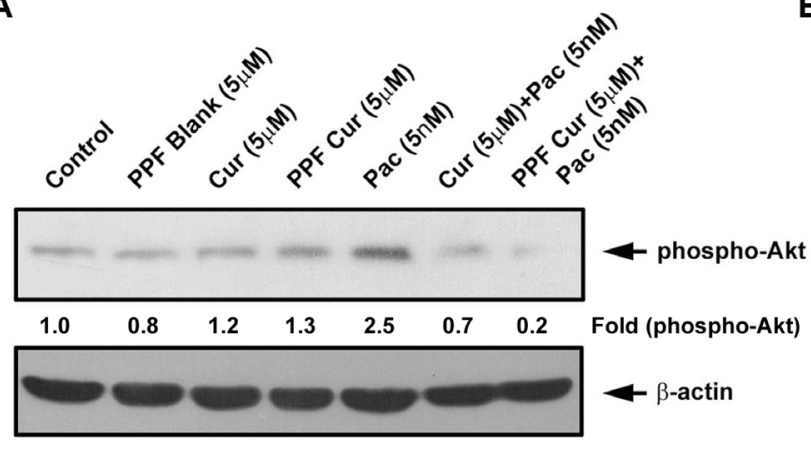

B

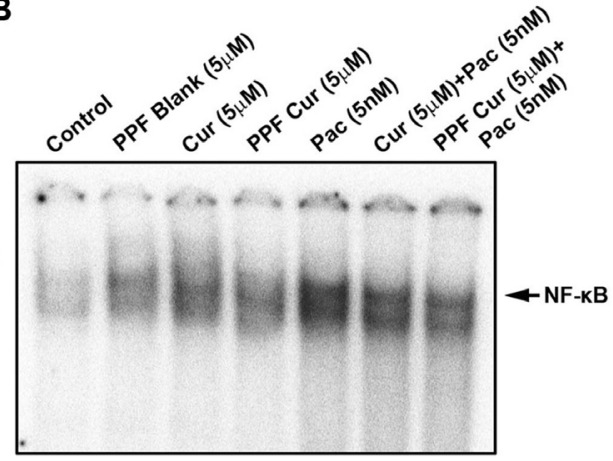

C

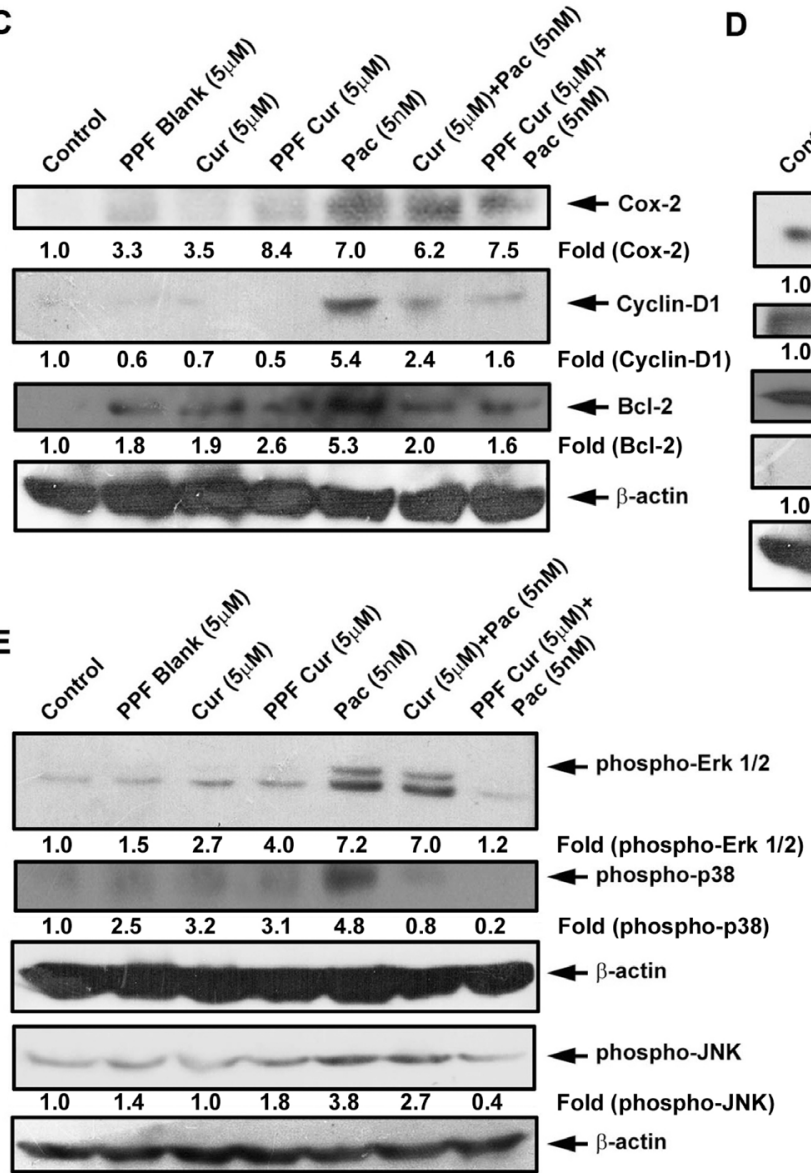

D
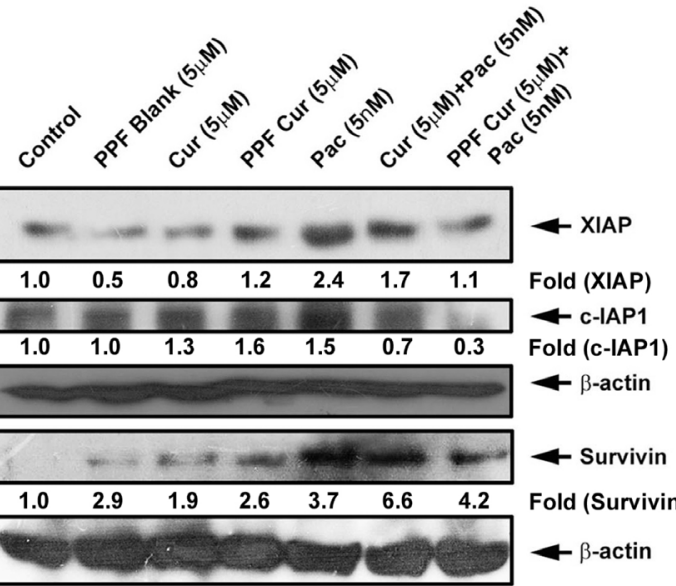

$\mathbf{F}$

Figure 2: Paclitaxel-induced activation of survival signals is down-regulated more efficiently by PPF-curcumin compared to free curcumin. (A) Paclitaxel-induced phosphorylation of Akt is more efficiently down-regulated by PPF-curcumin than free curcumin. HeLa cells were treated with free curcumin or PPF curcumin either alone or in combination with paclitaxel for $1 \mathrm{~h}$ after pre-treating with curcumin/PPF-curcumin and Western blotting and ECL were performed. (B) PPF-curcumin induced significant inhibition of NF-kB activation. HeLa cells were treated with free curcumin or PPF-curcumin for $2 \mathrm{~h}$ and/or paclitaxel for 30 min after pre-treating with curcumin/PPF-curcumin, after which nuclear extracts were prepared and EMSA was performed. (C) Paclitaxel-induced up-regulation of Cox-2, Cyclin-D1 and Bcl-2 are down-regulated more efficiently by PPF-curcumin than free curcumin. HeLa cells were treated with free curcumin or PPF-curcumin either alone or in combination with paclitaxel for $24 \mathrm{~h}$, after pre-treating with curcumin/PPF-curcumin and Western blotting and ECL were performed. (D) Paclitaxel-induced up-regulation of IAPs is more proficiently down-regulated by PPF-curcumin than free curcumin. HeLa cells were treated with free curcumin or PPF-curcumin either alone or in combination with paclitaxel for $24 \mathrm{~h}$, after pre-treating with curcumin/PPF-curcumin and Western blotting and ECL were performed. (E) Paclitaxel-induced phosphorylation of Erk 1/2, p38 and JNK are more effectively down-regulated by PPF-curcumin compared to free curcumin. HeLa cells were treated with free curcumin or PPF-curcumin and/or paclitaxel for $15 \mathrm{~min}$, after pre-treating with curcumin/PPF-curcumin and subjected to Western blotting followed by detection by ECL. All the blots were quantified and band density of individual bands are indicated in the blots. (F) PPF-curcumin induced significant inhibition of AP-1 activation. HeLa cells were treated with free curcumin or PPF-curcumin for $2 \mathrm{~h}$ and/or paclitaxel for $30 \mathrm{~min}$ after pre-treating with curcumin, after which nuclear extracts were prepared and EMSA was performed. 
curcumin alone did not significantly reduced the tumor growth, combination of curcumin or PPF-curcumin with paclitaxel significantly suppressed tumor growth, the latter being more efficient. The tumor volume of all the treatment groups is shown in Figure 4B and indicates the enhanced chemosensitizing efficacy of PPF-curcumin in paclitaxel chemotherapy. The $p$ value $(p$-value $\leq 0.001)$ indicates the statistical significance between control/ blank and lip cur+pac/PPF+Pac combination in the 4th week. We presume that the enhanced tumor reduction in animals treated with PPF-curcumin might have been due to enhanced intra-tumoral retention of curcumin in the case of PPF-curcumin. Further, we performed the immunohistochemical analysis for the expression levels of proliferating cell nuclear antigen (PCNA), NF- $\kappa \mathrm{B}$ subunit p65, pro-survival cyclin-D1 and VEGF. In animals administered with paclitaxel-PPF curcumin combination, PCNA, p65, cyclin-D1 and VEGF showed relatively low expression in tumor sections of human cervical cancer xenografts as shown in Figure 4C.

\section{PPF-curcumin shows enhanced tissue retention and serum concentration compared to free curcumin}

As both our in vitro and in vivo results have clearly established that PPF-curcumin is superior to liposomal curcumin in chemosensitizing cervical cancer, our next attempt was to study whether folic acid conjugation has augmented the tissue retention of curcumin in cervical tissues. We compared the tissue level uptake of liposomal curcumin and PPF-curcumin in the cervix of female Swiss albino mice. PPF-curcumin exhibited qualitative profound fluorescence in the cervix of healthy Swiss albino mice from $3 \mathrm{~h}$ onwards and fluorescence was visible up to $24 \mathrm{~h}$, while there was no detectable fluorescence in the tissue of mice treated with liposomal curcumin after $3 \mathrm{~h}$ as shown in Figure 5A. This might have been probably due to folic acid mediated targeting of PPFcurcumin towards FOLR1 in the cervix tissues [32]. We also recorded curcumin fluorescence in the serum
A
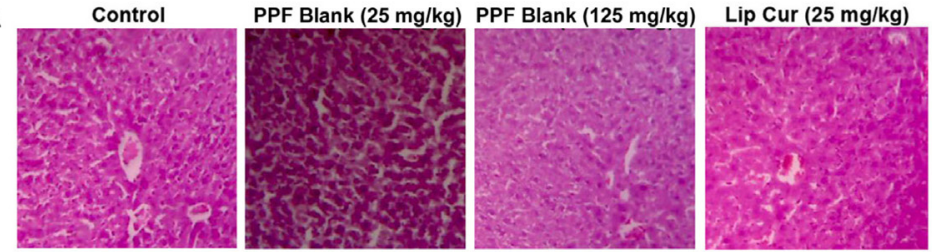

B
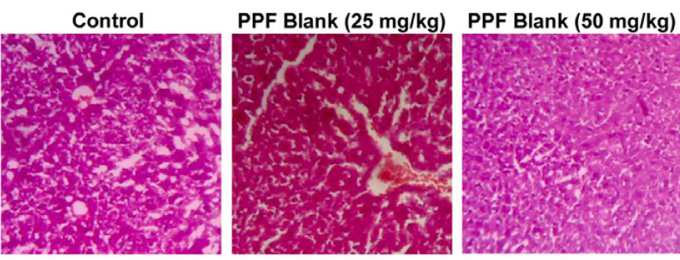

C

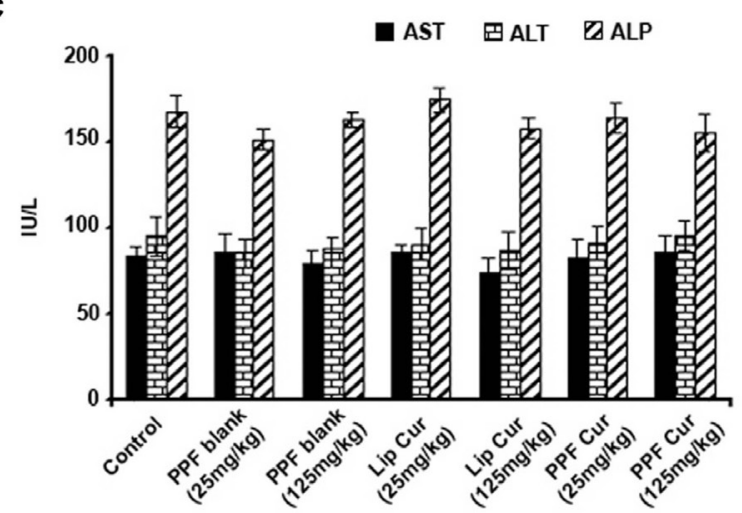

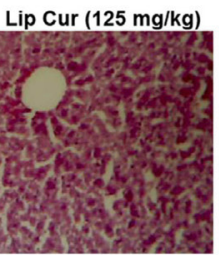
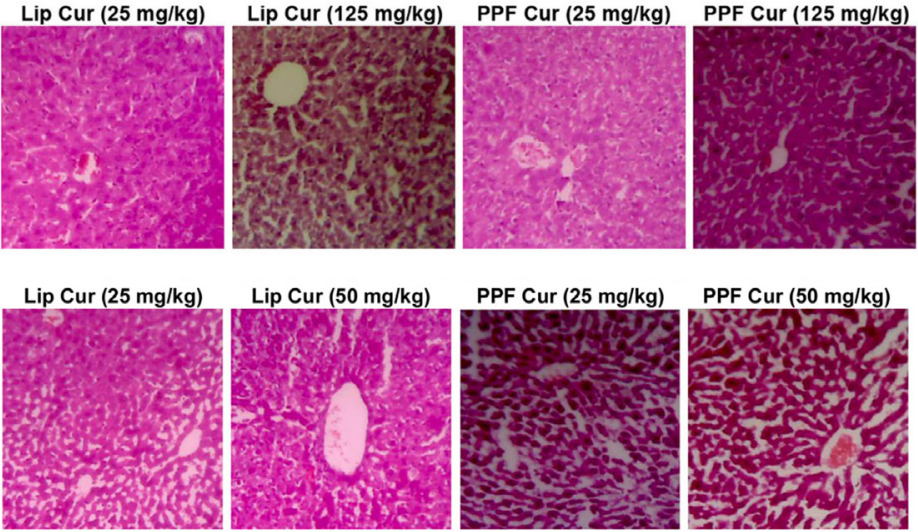

D

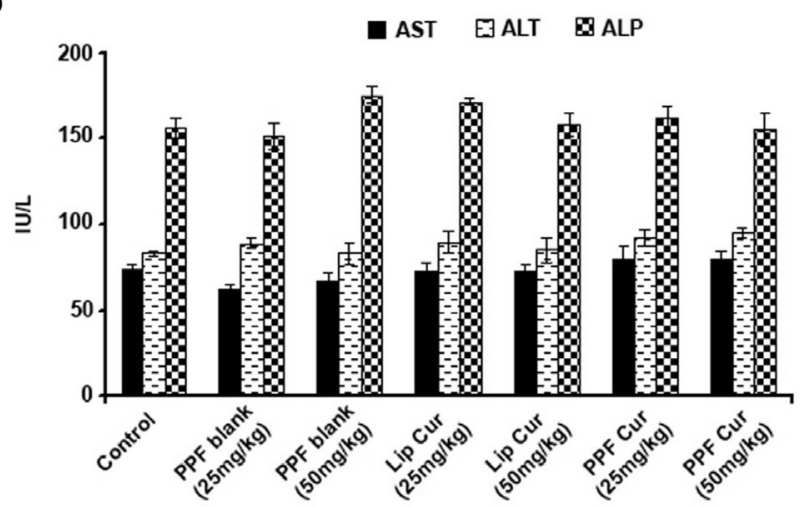

Figure 3: PPF curcumin is pharmacologically safe as assessed by acute and chronic toxicity studies in Swiss albino mice. (A) Histopathological analysis of liver tissues of mice administered with $25 \mathrm{mg} / \mathrm{kg}$ or $125 \mathrm{mg} / \mathrm{kg}$ liposomal curcumin, PPF-curcumin or the void carrier, during acute toxicity study (7 days). (B) Histopathological analysis of the liver tissues of mice subjected to chronic toxicity study for 2 months using $25 \mathrm{mg} / \mathrm{kg}$ or $50 \mathrm{mg} / \mathrm{kg}$ liposomal curcumin, PPF-curcumin or void carrier. (C) Liver function parameters of mice subjected to acute toxicity study using $25 \mathrm{mg} / \mathrm{kg}$ or $125 \mathrm{mg} / \mathrm{kg}$ liposomal curcumin, PPF-curcumin or the void carrier. (D) Liver function parameters of mice subjected to chronic toxicity study using $25 \mathrm{mg} / \mathrm{kg}$ or $50 \mathrm{mg} / \mathrm{kg}$ liposomal curcumin, PPF-curcumin or the void carrier. 
of mice using spectroflourimetry to study the extent of curcumin retention in vivo. Serum was isolated from mice administered with liposomal curcumin or PPF-curcumin and curcumin fluorescence was recorded. Figure 5B shows that, even after $12 \mathrm{~h}$ there was significant curcumin fluorescence in the serum of animals injected with 100 $\mathrm{mg} / \mathrm{kg}$ body weight of PPF-curcumin, whereas in the case of liposomal curcumin, there was considerable drop in the fluorescence intensity of curcumin after $6 \mathrm{~h}$. $p$ values $(p \leq 0.001)$ indicates the statistical significance for the same. Thus, the imaging studies and spectrofluorimetry studies clearly depict that, PPF-curcumin enables considerable uptake, improved tissue retention and prolonged circulation of curcumin in vivo.

\section{Folate receptor specific curcumin delivery} using folic acid conjugated PLGA-PEG nanoparticles significantly improves the cellular uptake of curcumin, which in turn enhances its chemosensitizing potential

Our next endeavor was to investigate whether the enhanced chemosensitizing efficacy of PPF-curcumin can be correlated to the over-expression of FOLR1 in HeLa cells. As already reported [19], HeLa cells exhibited high expression of FOLR1 as indicated in Figure 6A. The HeLa xenografts also exhibited very strong expression of FOLR1 as shown in Figure 6B. In contrast, the non-tumorigenic immortalized $\mathrm{HaCaT}$ cells did not display significant

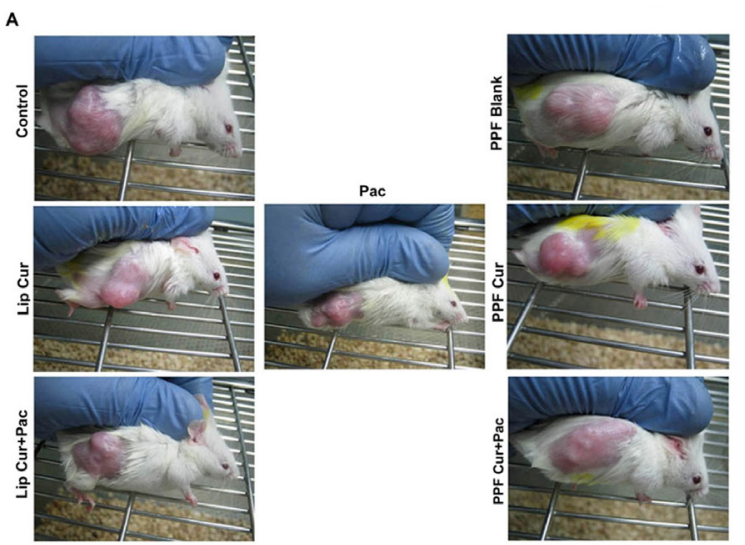

C
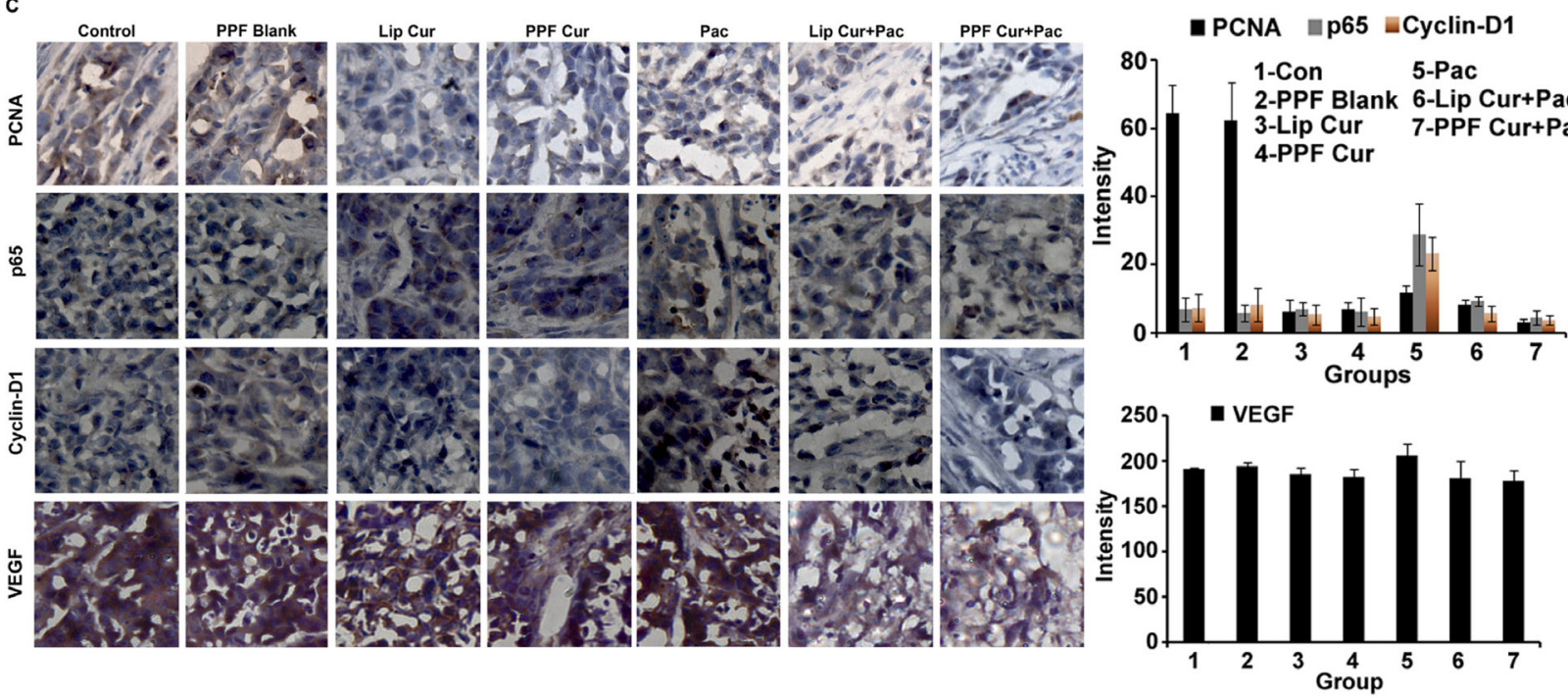

Figure 4: PPF-curcumin causes significant sensitization of HeLa xenograft tumors in NOD-SCID mice to paclitaxel treatment. (A) Representative images of NOD-SCID mice bearing HeLa xenograft tumors, after 4 weeks of treatment. (B) Graph showing tumor volumes of NOD-SCID mice bearing HeLa xenograft tumors with or without treatment during the span of study. Combination treatment significantly ( $p$-values $\leq 0.001)$ reduced the tumor volume in comparison to control and blank treated mice. $(\mathbf{C})$ Immunohistochemical staining of PCNA, p65, cyclin D1 and VEGF in the tumor tissues of different treatment groups, which shows that PPF-curcumin efficiently down-regulates paclitaxel-mediated activation of survival signals in HeLa xenograft tumors in NOD-SCID mice. The quantification of the IHC images were done using ImageJ software. The results of three independent data sets are compiled as graphs. The values shown are average \pm SD for 3 independent images/fields. 
expression of FOLR1 as represented in Figure 6C. So we presumed that, the difference in cytotoxicity induced by PPF-curcumin in HeLa and HaCaT may be due to the differential expression of FOLR1 causing significantly enhanced FOLR1-mediated endocytosis and cytosolic retention of curcumin in HeLa cells in a receptor targeted fashion. Attesting our assumption, HeLa cells revealed considerable internalization of curcumin when treated with PPF-curcumin as shown in Figure 6D. Our hypothesis was further confirmed, when we could not find a significant internalization of curcumin in $\mathrm{HaCaT}$ cells (Figure 6E), which had minimal FOLR1 expression (Figure 6C). To confirm this inference, we pre-treated HeLa cells with folic acid before treating with PPF-curcumin and compared the fluorescence of curcumin within the cells. Figure $6 \mathrm{~F}$ indicates that pre-treatment of the cells with free folic acid drastically reduced the uptake of PPF-curcumin in HeLa cells, which have a very high expression of FOLR1. Further, we also compared the efficacy of PPF-curcumin in chemosensitizing HeLa cells towards paclitaxel, in the presence and absence of free folic acid. Interestingly, the synergism was completely lost in the presence of free folic acid as shown in Figure 6G. However, as can be seen from the Figure $6 \mathrm{G}$, free folic acid did not significantly enhance the cell viability in PPF-curcumin/paclitaxel combination. This may be because even though free folic acid quench the folate receptors, paclitaxel can still exhibit its cytotoxic activity. Moreover, the quenching of folate receptors by free folic acid is transient and hence PPFcurcumin can exert its chemosensitizing activity thereafter. To substantiate these results in vivo, we compared the chemosensitizing efficacy of PPF-curcumin with that of PLGA-curcumin, which lack folic acid conjugation, using HeLa xenograft model in NOD-SCID mice. Though both the formulations exhibited chemosensitizing potential, that brought about by PPF-curcumin was significantly better as indicated by Figure $6 \mathrm{H}$. We compared the NF- $\kappa \mathrm{B}$ and AP-1 status in various groups as our previous study had demonstrated key regulatory roles for these molecules in modulating the synergism of curcumin and paclitaxel [10]. Interestingly, PPF-curcumin induced a drastic inhibition in paclitaxel-induced DNA binding of NF- $\kappa$ B and AP-1 in the tumor tissues as shown by Figure 6I. The band intensities were quantified and represented in Supplementary Table 2. PLGA-curcumin also inhibited both the molecules, though to a lesser extent, demonstrating that folic acid conjugation to PLGA-curcumin nanoparticles significantly improves its chemosensitizing potential.
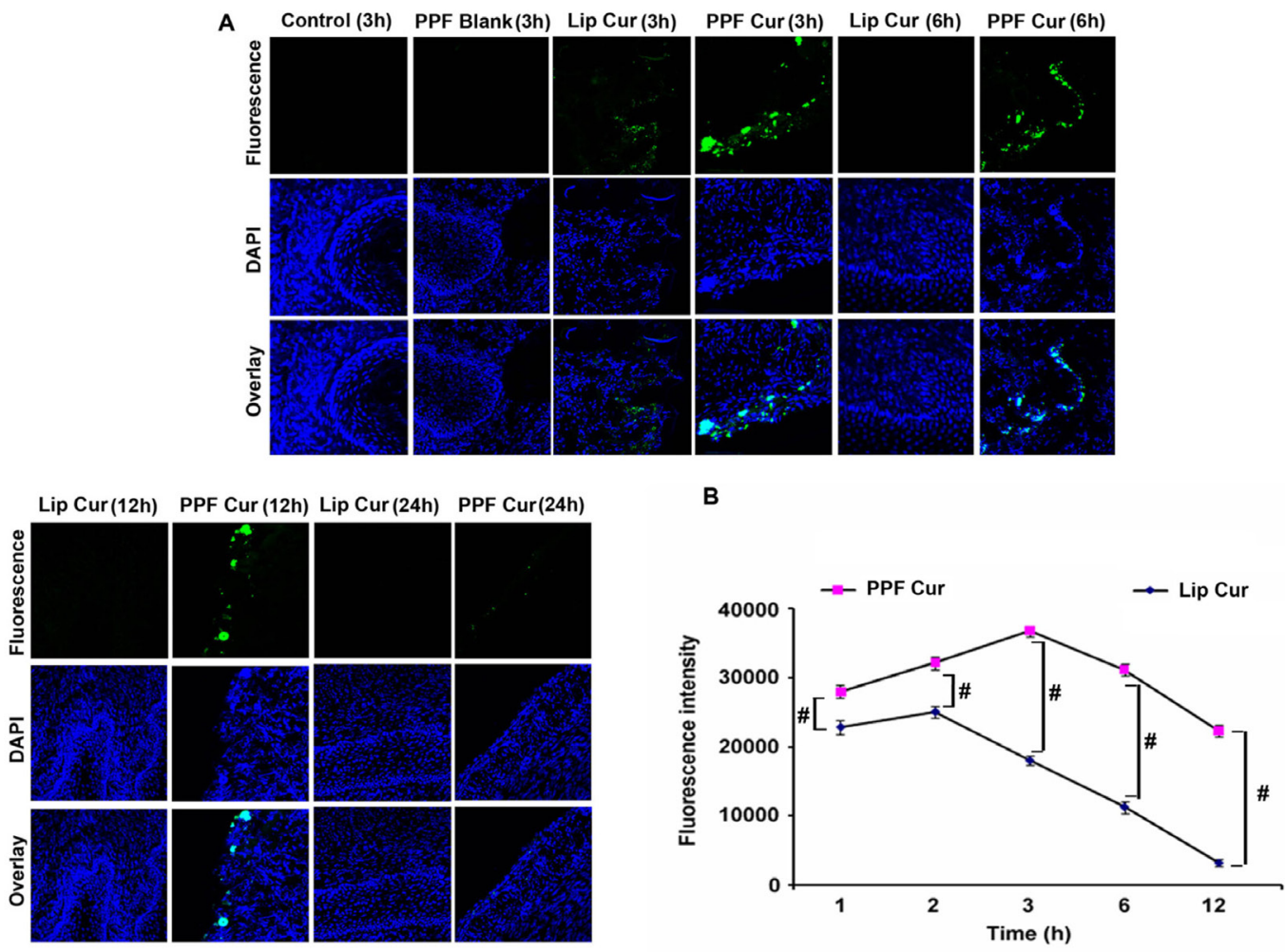

Figure 5: PPF-curcumin exhibits enhanced bioavailability and tissue retention of curcumin. (A) PPF encapsulation increases the retention time of curcumin in cervical tissues. Mice were intraperitoneally injected with liposomal curcumin or PPF-curcumin $(100 \mathrm{mg} / \mathrm{kg})$ and intracellular fluorescence for curcumin in cervical tissues were assessed by confocal microscopy. Nuclei were stained with DAPI and images were captured at a magnification of 60X. (B) PPF-curcumin exhibits enhanced circulation of curcumin compared to liposomal curcumin. Mice were intraperitoneally injected with liposomal curcumin or PPF-curcumin (100 mg/kg). The serum was isolated and fluorescence was measured at different time intervals. \#indicates $p \leq 0.001$. 


\section{DISCUSSION}

Though paclitaxel is a widely used anti-cancer agent [33], the severity of side-effects of this drug and the solvent used for its parenteral administration are major factors that hamper its therapeutic outcome [34]. Prolonged administration of chemodrugs including paclitaxel has been shown to switch on several survival signals such as NF-kB, Akt, MAPK and AP-1, ultimately leading to chemoresistance [35]. Curcumin has been established as a successful chemosensitizer capable of down-regulating the expression of proliferation, anti-apoptotic and metastatic gene products ultimately resulting in the reversal of chemoresistance [36-38]. Both in vitro and in vivo studies conducted earlier in our laboratory have demonstrated the efficacy of curcumin in sensitizing cervical cancer cells towards paclitaxel, wherein significant down-regulation of several survival pathways was observed, though NF$\kappa \mathrm{B}$ was the key regulatory molecule $[10,11]$. For both the in vivo tumor models, we had administered curcumin in a liposomal formulation [11]. The major drawbacks of this formulation were poor bioavailability and low tissue retention of curcumin. To overcome these limitations, we synthesized various formulations of curcumin and studied their physico-chemical characteristics and therapeutic efficacy, in vitro [17-19, 39]. Although none of these formulations exhibited drastic improvement in the anti-cancer potential than free curcumin, it should be

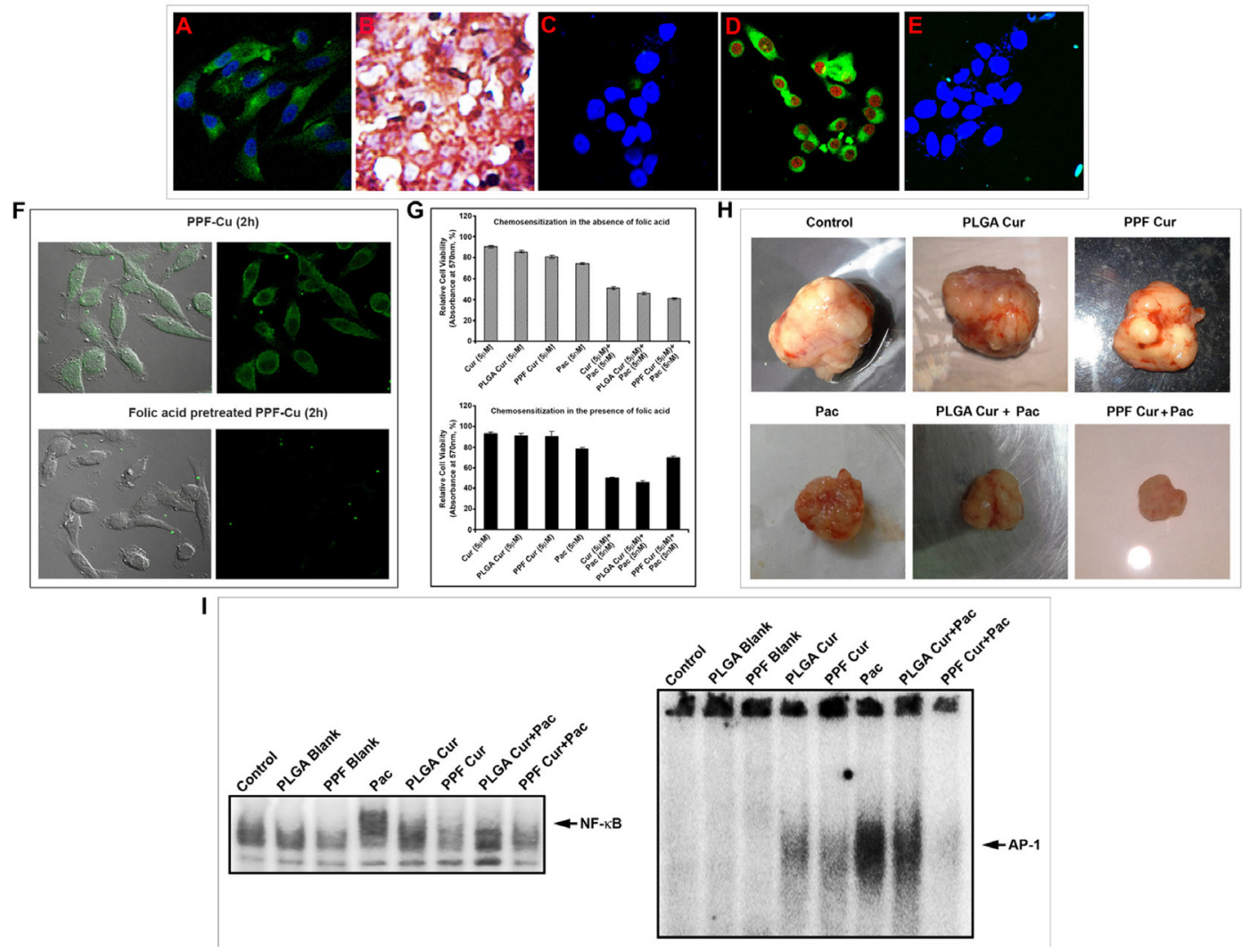

Figure 6: Folic acid conjugation is responsible for the enhanced chemosensitization potential of PPF-curcumin. (A) Confocal microscopy image showing high expression of FOLR1 in HeLa cells. (B) Immunohistochemical staining of FOLR1 expression in HeLa xenografts. (C) Confocal microscopy image showing low expression of FOLR1 in HaCaT cells. (D) Uptake of PPF-curcumin nanoparticles in FOLR1 over-expressing HeLa cells. Cells were incubated with $5 \mu \mathrm{M}$ PPF-curcumin nanoparticles for $2 \mathrm{~h}$. Cells were fixed and nuclei were stained with PI and images were captured at a magnification of 40X. (E) Confocal microscopy image showing the reduced uptake of PPF-curcumin in HaCaT cells. Cells were incubated with $5 \mu \mathrm{M}$ PPF-curcumin nanoparticles for $2 \mathrm{~h}$. Cells were fixed and nuclei were stained with DAPI and images were captured at a magnification of 40X. (F) Uptake of PPF-curcumin in HeLa cells in the absence or presence of free folic acid. Reduced uptake of PPF-curcumin was observed in HeLa cells in the presence of free folic acid indicating FOLR1-targeted uptake of PPF-curcumin in FOLR1 over-expressing HeLa cells. Cells were incubated with free folic acid for 20 min under culture conditions as described in Materials and Methods. Later, the medium was removed and incubated with $5 \mu \mathrm{M}$ PPFcurcumin nanoparticles for $2 \mathrm{~h}$. For confocal microscopy, cells were fixed and nuclei were stained with DAPI and images were captured at a magnification of 40X. (G) Chemosensitization studies in HeLa cells using curcumin, PLGA-curcumin or PPF-curcumin in the presence or absence of folic acid. For cytotoxicity study, cells were cultured in the presence of $100 \mu \mathrm{g} / \mathrm{ml}$ and treated with curcumin or PLGA-curcumin or PPF-curcumin $(5 \mu \mathrm{M})$ either alone in combination with paclitaxel $(5 \mathrm{nM})$. Later, after $72 \mathrm{~h}$ of incubation, the cell viability was assessed using MTT. (H) Representative images of tumors from NOD-SCID mice bearing HeLa xenograft tumors with or without treatment after 4 weeks. (I) PPF-curcumin induced significant inhibition of NF-kB and AP-1 activation in vivo as assessed by EMSA. 
mentioned that all these formulations were administered to the in vitro systems in aqueous dispersible suspensions, while free curcumin was administered in DMSO, which is an undesirable vehicle for in vivo applications. Thus, we could overcome one of the major hurdles pertaining to the hydrophobic nature of curcumin, which significantly hamper the intracellular delivery of the compound. Our next attempt was to prepare a formulation, which will help us to deliver curcumin in a target-specific manner. We selected PLGA nanoparticles as vehicle to deliver curcumin for our in vivo study, since PLGA is a widely accepted non-toxic material approved by FDA for human applications [17, 19]. Moreover the biodegradable nature of PLGA makes it attractive for in vivo applications [40-42]. Since FOLR1 is widely over-expressed in almost all cancer cells, we selected folic acid as our tumor targeting ligand. Several groups have reported the conjugation of nanoparticles with folic acid for tumor targeted nanoparticle delivery [43-45]. We conjugated folic acid to curcumin-encapsulated PLGA-PEG copolymer nanoparticles (PPF-curcumin) and compared its efficacy as a chemosensitizer for paclitaxel chemotherapy of cervical cancer, with that of liposomal curcumin [11]. Since it has been reported that, liposome formulations are internalized in cancer cells in a non-targeted fashion, which can cause curcumin accumulation in undesirable sites [46, 47], we performed a detailed investigation to evaluate and compare the chemosensitization potential of both the formulations using both in vitro and in vivo experiments. The enhanced chemosensitization potential of PPF-curcumin was evident when it induced significant enhancement in paclitaxel- mediated apoptosis and inhibition of clonogenic potential of HeLa cells compared to free curcumin. It was also interesting to see that the formulation did not chemosensitize the non-tumorigenic immortalized HaCaT cells towards paclitaxel. Moreover, the in vivo acute and chronic toxicity studies, confirmed that PPF-curcumin is pharmacologically safe either alone or in combination with paclitaxel. At the same time, the formulation brought about considerable augmentation in the efficacy of curcumin in down-regulating the pivotal molecules regulating the synergistic toxicity of paclitaxel and curcumin towards cervical cancer cells [10]. In conjunction with the in vitro studies, PPF-curcumin showed enhanced chemosensitization potential towards paclitaxel in cervical cancer xenograft models of NODSCID mice, than liposomal curcumin, causing significant tumor reduction in vivo. The tumor reduction was supported by the results of various molecular assays and immunohistochemical analyses, which indicate that PPFcurcumin in combination with paclitaxel is exceedingly successful in down-regulating the key survival pathways responsible for the uncontrolled proliferation, apoptosis evasion and tumor progression. Thus, the success of PPF-curcumin over liposomal curcumin in downregulating the survival signals induced by paclitaxel can be attributed to its FOLR1 targeting capability, which was successfully substantiated by the drastic difference in FOLR1 expression and curcumin uptake in both $\mathrm{HeLa}$ and $\mathrm{HaCaT}$ cells, where the latter possess minimal FOLR1. This observation is further authenticated when; uptake of PPF-curcumin in FOLR1 over-expressing HeLa cells was drastically reduced by free folic acid pretreatment. The low expression of FOLR1 and hence the low uptake of PPF-curcumin in HaCaT cells may be the reason behind the absence of synergistic cytotoxicity of paclitaxel-curcumin combination, even after delivering curcumin as PPF-curcumin. The results of both in vitro cytotoxicity studies and xenograft tumor reduction studies, which compared the chemosensitizing efficacy of PLGA-curcumin and PPF-curcumin towards paclitaxel chemotherapy of cervical cancer clearly demonstrated and confirmed that folic acid conjugation is the modification responsible for the extra efficacy of PPF-curcumin. Our previous study has shown that, Akt is upstream and MAPKs are downstream of NF- $\kappa \mathrm{B}$, which act as a key regulator of curcumin-mediated chemosensitizion of paclitaxel chemotherapy of cervical cancer [10]. AP-1 is a transcription factor, which is stimulated by a complex network of MAPKs of ERK, p-38 and JNK families [11]. Corroborating this fact, PPF-curcumin caused significantly better abrogation of NF- $\kappa \mathrm{B}$ and AP-1, compared to PLGAcurcumin, which may be correlated with the extent of tumor reduction observed in the animals treated with PPF-curcumin. Thus in the current study, we have clearly demonstrated how folic acid conjugation to curcuminencapsulated PLGA-PEG nanoparticles significantly improves the bioavailability and tissue retention of curcumin, thereby enhancing the efficacy of curcumin as a chemosensitizer in FOLR1 over-expressing cervical cancer cells. The proposed mechanism of action by which PPF-curcumin causes the suppression of survival signals, which leads to the chemosensitization of cervical cancer cells towards paclitaxel, is illustrated in Figure 7. PPF-curcumin is internalized into the cancer cells via FOLR1-mediated endocytosis. In the cytosol, curcumin is released due to the degradation of PLGA nanoparticles aided by the acidic microenvironment, which further down-regulates various survival signals up-regulated by paclitaxel ultimately causing the cells to undergo apoptotic cell death.

The current study gains immense significance in the scenario of accumulating evidences of chemoresistance induced by prolonged exposure of cancer cells to chemotherapeutics. Therefore, with regard to future clinical applications, the present strategy may offer promising therapeutic benefits that may be achieved at low concentrations of paclitaxel, which in turn may overcome chemoresistance and toxic side-effects, the major hurdles impeding the clinical outcome of several anti-cancer drugs. 


\section{MATERIALS AND METHODS}

\section{Cell lines}

$\mathrm{HeLa}$ and $\mathrm{HaCaT}$ cells were procured from National Centre for Cell Sciences (NCCS), Pune, India.

\section{Chemicals}

Curcumin, antibody against $\beta$-actin, and the horseradish peroxidase (HRP) conjugated secondary antibodies were obtained from Sigma-Aldrich (St. Louis, USA). Curcumin with a purity of $\geq 65 \%$ (HPLC) was used for our studies. Paclitaxel was obtained from Calbiochem (San Diego, CA, USA). MTT was purchased from Calbiochem
(La Jolla, USA). DAPI (4',6-Diamidino-2-phenylindole dihydrochloride), antibodies against Cox-2, PARP and Cyclin-D1 were purchased from Santacruz Biotechnology (Santa Cruz, CA, USA). The radio labelled [ $\left.{ }^{\gamma_{-} 32} \mathrm{P}\right]$ ATP was obtained from Bhabha Atomic Research Centre (BARC), India. The oligonucleotide probes used for electrophoretic mobility shift assays were custom synthesized (Genosys, Sigma). Dulbecco's Modified Eagle Medium (DMEM) and streptomycin sulphate were purchased from Invitrogen Corporation (Grand Island, USA). Antibodies against p-Akt, p-p42/44, p-JNK, p-P38 and monoclonal antibody against caspase-3 and rabbit polyclonal antibody against caspase- 9 were purchased from Cell Signaling Technologies (Beverly, MA, USA). Immobilon Western Chemiluminescent HRP Substrate was purchased from Millipore (Billerica, MA,

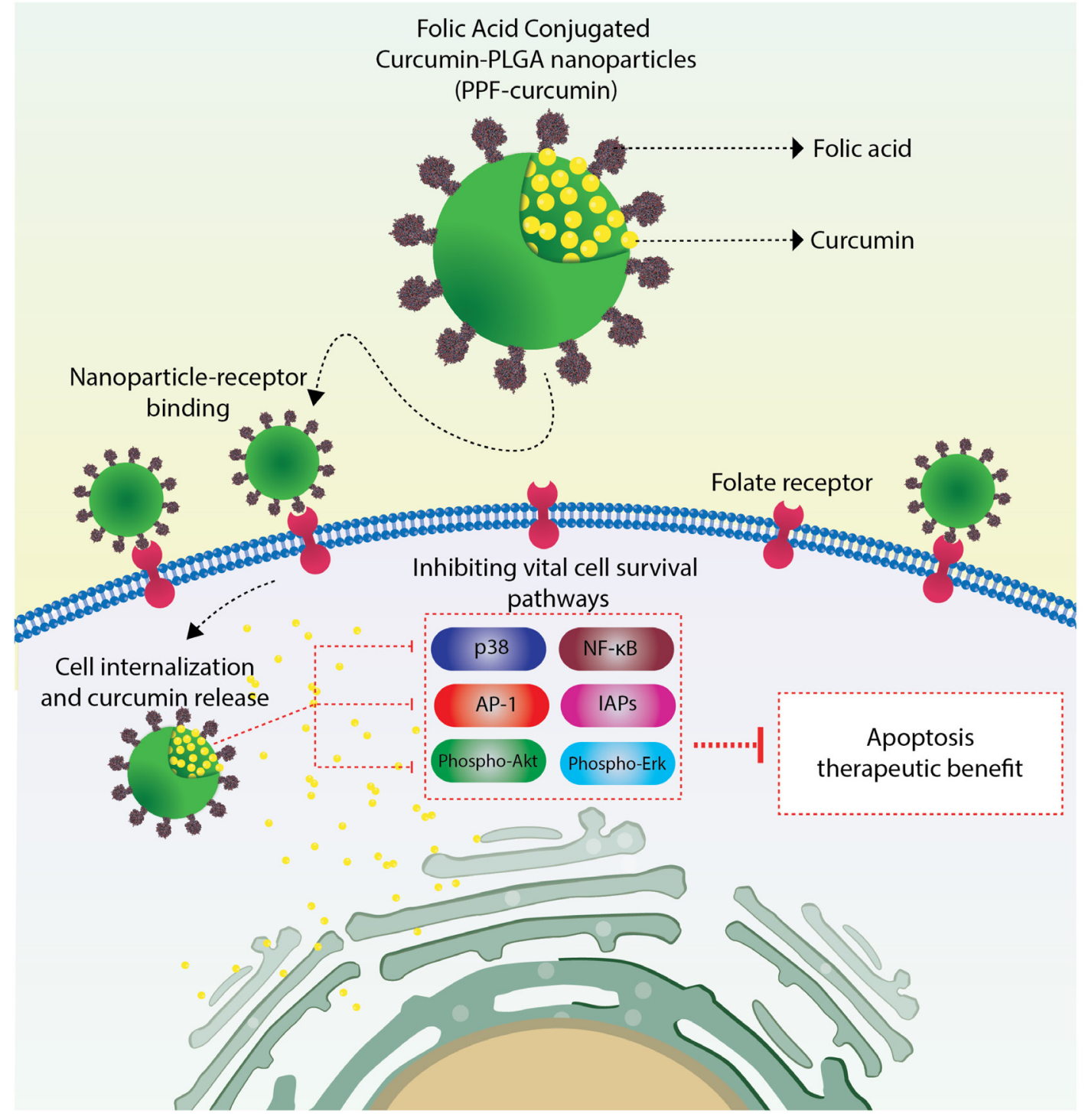

Figure 7: Pictorial representation of the mechanism of action of PPF-curcumin. PPF-curcumin is internalized into the cancer cells via FOLR1-mediated endocytosis. In the cytosol, curcumin is released due to the degradation of PLGA nanoparticles aided by the acidic microenvironment, which further down-regulates various survival signals (p-38, NF- $\mathrm{B}$, AP-1, IAPs, phospho-Akt, phospho-Erk up-regulated by paclitaxel ultimately causing the cells to undergo apoptotic cell death. 
USA). Super Sensitive ${ }^{\mathrm{TM}}$ Polymer-HRP Detection System kit was procured from Biogenex (Fremont, CA, USA). Antibody against folate receptor (FOLR1 antibody) was purchased from Invitrogen Corporation (Grand Island, USA). All other reagents were procured from Sigma-Aldrich, unless otherwise mentioned.

\section{Synthesis and characterization of PLGA- curcumin and PPF-curcumin}

Synthesis of PLGA-curcumin and PPF-curcumin was performed as reported earlier [19]. Poly lactic-coglycolic acid (PLGA) $50: 50$ (lactic/glycolic ratio) having MW $24000 \mathrm{Da}$ to $38000 \mathrm{Da}$, poly ethylene glycol (PEG) bisamine MW 3000 Da, N-hydroxy succinimide (NHS) MW 115.09, dicyclohexylcarbodiimide (DCC) MW 206.3, pyridine, folic acid, dichloro methane (DCM), dimethyl sulfoxide (DMSO), acetone, curcumin, acridine orange and ethidium bromide were procured from Sigma (Steinheim, Germany). Polyvinyl alcohol (PVA) 87\% hydrolysed was purchased from SD fine (Mumbai, India). For morphological analysis of PPF-curcumin using transmission electron microscope (TEM, JEOL 1011, Japan), nanoparticle suspension was diluted in Milli- ${ }^{\circledR}$ (Millipore Corporation, Billerica, MA) water at $25^{\circ} \mathrm{C}$ and drop casted onto formvar coated grids.

\section{Drug treatment}

Cells were pretreated with curcumin or PPFcurcumin $(5 \mu \mathrm{M})$ either alone or in combination with paclitaxel $(5 \mathrm{nM}), 2$ hours prior to paclitaxel $(5 \mathrm{nM})$ treatment. The DMSO concentration in all the experiments was maintained $<0.25 \%$.

\section{MTT assay}

Cell viability after drug treatment was determined by MTT assay. Cells $\left(\sim 2.5 \times 10^{3}\right)$ seeded in 96-well plate were treated with drugs either alone or in combination and MTT assay was performed as described earlier [11], after 72 hours of incubation, post paclitaxel treatment. Untreated cells, PPF and PLGA blank treated cells were used as controls.

\section{Clonogenic assay}

To compare the anti-clonogenic potential of different formulations of curcumin either alone or in combination with paclitaxel clonogenic assay was performed as reported earlier [19].

\section{Western blot analysis}

The whole cell lysate was prepared from the cells treated with or without drug and subjected to Western blot analysis as reported earlier [19].

\section{Chromatin condensation assay}

Chromatin condensation assay was performed in drug treated cells after 24 hours of post drug treatment followed by fixing the cells and staining the nucleus using DAPI. The stained cells were mounted on a glass slide using Flouromount ${ }^{\mathrm{TM}}$ aqueous mounting medium (Sigma Aldrich, USA) and images were taken using a fluorescent microscope.

\section{Cellular uptake of PPF-curcumin in HeLa cells}

Briefly, $2 \times 10^{4}$ cells were grown on cover slips placed in 24 well plates. Once the cells attained $80 \%$ confluency, they were treated with $5 \mu \mathrm{M}$ PPF-curcumin for $2 \mathrm{~h}$. The cells were washed with PBS and fixed using $4 \%$ paraformaldehyde. The nuclei were stained using propidium iodide for 5 min and were mounted using DPX. Cells were then examined for intracellular fluorescence of curcumin using confocal laser scanning microscope in the FITC channel $(488 \mathrm{~nm})$ and images were captured at $40 \mathrm{X}$.

\section{Cellular uptake of PPF-curcumin in HaCaT cells}

Briefly, $2 \times 10^{4}$ cells were grown on cover slips placed in 24 well plates and treated with $5 \mu \mathrm{M}$ PPFcurcumin for $2 \mathrm{~h}$. The cells were washed with PBS and fixed using $4 \%$ paraformaldehyde. The nuclei were stained using $1 \mu \mathrm{g} / \mathrm{ml}$ DAPI for $10 \mathrm{~min}$ and were mounted using DPX. Cells were then examined for intracellular fluorescence of curcumin using confocal laser scanning microscope in the FITC channel (488 nm) and images were captured at $40 \mathrm{X}$.

\section{FOLR1 quenching studies using confocal microscopy}

Approximately, $2 \times 10^{4}$ cells were grown on cover slips placed in 24 well plates. Later, they were incubated with PPF-curcumin with or without folic acid pretreatment (100 $\mu \mathrm{l}$ of $4 \mathrm{mg} / \mathrm{ml}$ folic acid for $20 \mathrm{~min}$ ), which has earlier proved to quench FOLR1 [48-50]. Later, the medium was removed, rinsed with PBS, fixed using $4 \%$ paraformaldehyde, stained with DAPI and observed at 40X under confocal microscope using $488 \mathrm{~nm}$ laser to record curcumin fluorescence.

\section{Cell viability of HeLa cells with and without folic acid pre-treatment}

Approximately, $\sim 2.5 \times 10^{3}$ cells were seeded in 96-well plate and treated with curcumin or PLGAcurcumin or PPF-curcumin $(5 \mu \mathrm{M})$ either alone in combination with paclitaxel $(5 \mathrm{nM})$ in the presence or absence of free folic acid $(100 \mu \mathrm{g} / \mathrm{ml})$. Later, after $72 \mathrm{~h}$ of incubation, the cell viability was assessed using MTT cell viability assay as described earlier. 


\section{Confocal microscopy for FOLR1 staining}

$\mathrm{HeLa}$ or HaCaT cells were seeded on coverslips at a density of 5000 cells per well in a 24 well cell culture plate. Later, the cells were fixed using $4 \%$ paraformaldehyde and incubated with rabbit polyclonal antibody against FOLR1 at $4-8^{\circ} \mathrm{C}$, followed by FITC conjugated secondary antibody. Later, the cells were washed and stained with DAPI and observed at $40 \mathrm{X}$ under confocal microscope using $488 \mathrm{~nm}$ laser.

\section{In vivo studies}

The methods were carried out in accordance with the Committee for the Purpose of Control and Supervision of Experiments on Animals guidelines and all experimental protocols were approved by Institutional Animal Ethical Committee of Rajiv Gandhi Centre for Biotechnology.

\section{Toxicity studies}

Acute (7 days) and chronic (60 days) toxicity studies were carried out in female Swiss albino mice, 6 weeks of age. For acute toxicity study, liposome curcumin, PPF-curcumin $(25 \mathrm{mg} / \mathrm{kg}$ and $125 \mathrm{mg} / \mathrm{kg}$, 5 mice per group) either alone or in combination with paclitaxel in cremophor $(10 \mathrm{mg} / \mathrm{kg}, 5$ mice per group) or their corresponding void carriers were administered through intraperitonial injection. The mice were observed continuously for $1 \mathrm{~h}$ for any gross behavioral changes and death, and intermittently for the next $6 \mathrm{~h}$ and $24 \mathrm{~h}$ after dosing over a period of 7 days. For chronic toxicity study, liposome curcumin or PPF-curcumin $(25 \mathrm{mg} / \mathrm{kg}$ and $50 \mathrm{mg} / \mathrm{kg}, 5$ mice per group) either alone or in combination with paclitaxel in cremophor $(10 \mathrm{mg} / \mathrm{kg}$, 5 mice per group) or their corresponding void carriers were administered through intra-peritoneal injection (IP) every alternate day over a period of 14 days followed by a twice weekly regimen for 2 months. All mice were euthanized using $\mathrm{CO}_{2}$ at the end of the experiment, and blood was collected by cardiac puncture. Serum was isolated by centrifuging the coagulated blood samples at $1500 \mathrm{rpm}$ for $10 \mathrm{~min}$. Liver function parameters (AST/SGOT, ALT/SGPT) were recorded to assess the hepatotoxicity. The biochemical assays were conducted at Iype's Diagnostics, Thiruvananthapuram.

\section{In vivo bioavailability studies}

Female Swiss albino mice (6 weeks of age) were intraperitoneally injected with either liposomal curcumin or PPF-curcumin $(100 \mathrm{mg} / \mathrm{kg})$ or their void polymer carriers. The animals were sacrificed using $\mathrm{CO}_{2}$ after the desired time interval and blood was collected by cardiac puncture. Cervical tissues were collected, washed in PBS and were immediately cryosectioned without fixation. The tissue sections were stained using DAPI and mounted using
Flouromount ${ }^{\mathrm{TM}}$. The images were recorded using Nikon A1R confocal microscope at FITC channel $(488 \mathrm{~nm})$ and images were analyzed with NIS elements software.

\section{Tumor reduction studies using NOD-SCID mice bearing xenograft}

Tumor reduction studies were conducted as reported earlier [11]. Female NOD-SCID (NOD.CB17-Prkds ${ }^{\text {sid/s) }}$ mice of age 6-8 weeks were used for the experiment. Tumors were induced by subcutaneous injection of $\mathrm{HeLa}$ cells $\left(\sim 5 \times 10^{6}\right.$ cells in $100 \mu \mathrm{l} \mathrm{X}$ PBS $)$ in the lower right flank of mice and were allowed to grow for a period of time (approximately 15 days) to a size of approximately $100-150 \mathrm{~mm}^{3}$ as measured by vernier calipers. The animals were then randomly grouped into 9 groups of 5 each: Control, PLGA blank, PPF blank, liposomal curcumin, PPF-curcumin, paclitaxel, liposomal curcumin + paclitaxel, PLGA-curcumin+ paclitaxel and PPF-curcumin + paclitaxel group. Liposomal curcumin, PLGA-curcumin or PPFcurcumin (equivalent to $25 \mathrm{mg} / \mathrm{kg}$ curcumin, on alternate days) and paclitaxel (10 mg/kg doses twice weekly) were injected intraperitonially. Tumor volume was measured once in a week. Animals were sacrificed after 40-45 days and tumor samples were collected for further analyses.

\section{Histopathology and immunohistochemistry}

The mice tissues were collected and cryosectioned. Immunohistochemical analysis of various proteins in the xenograft tumor tissue sections was performed using the detection kit, as per manufacturer's protocol Super Sensitive Polymer-HRP IHC Detection System (Biogenex, CA, USA). The images were taken using Leica DM 1000 microscope. The results were interpreted by Dr. Sankar Sundaram, Deparatment of Pathology, Government Medical College Hospital, Kottayam.

\section{Electrophoretic mobility shift analysis}

Nuclear extracts from tissue samples or cultured cells were prepared and EMSA was performed to evaluate DNA-binding activity of NF-kB or AP-1 as described in already reported studies [11].

\section{Statistical analysis}

Data represent three independent sets of experiments. The error bars represent \pm S.D. Statistical analysis was undertaken using Prism 5.0c Software. A two-tailed $t$-test or a one-way ANOVA was performed when comparing two groups or more than two groups, respectively. Statistical significance was defined as $P<0.05$. Data are shown as mean $\pm \mathrm{SD}$. Two-way ANOVA followed by Tukey's post hoc $t$-test analysis was used for statistical comparison between different groups. "means $P \leq 0.001{ }^{*}$ means $P \leq 0.01$. 


\section{Author contributions}

Conception and design: Ruby John Anto, Arun Kumar T. Thulasidasan, Archana P. Retnakumari; Development of methodology: Ruby John Anto, Gopalakrishnapillai Sankaramangalam Vinod Kumar, Arun Kumar T. Thulasidasan, Archana P. Retnakumari; Acquisition of data: Arun Kumar T. Thulasidasan, Archana P. Retnakumari, Mohan Shankar, Vinod Vijayakurup, Shabna Anwar, Jisha J Pillai, Devika Nandan; Analysis and interpretation of data: Ruby John Anto, Gopalakrishnapillai Sankaramangalam Vinod Kumar, Sankar Sundaram, Arun Kumar T. Thulasidasan, Archana P. Retnakumari, Mohan Shankar, Vinod Vijayakurup; Writing, review and/or revision of the manuscript: Ruby John Anto, Arun Kumar T. Thulasidasan, Archana P. Retnakumari; Administrative, technical, or material support: Ruby John Anto, Gopalakrishnapillai Sankaramangalam Vinod Kumar, Arun Kumar T. Thulasidasan, Archana P. Retnakumari, Mohan Shankar, Vinod Vijayakurup, Shabna Anwar; Study supervision: Ruby John Anto; Other: Sanu Thankachan, Kavya S Pillai, Vijai V Alex and Teena Jacob Chirayil provided technical assistance.

\section{ACKNOWLEDGMENTS AND FUNDING}

This work was supported by DBT, Govt. of India (BT/PR10825/NNT/28/111/2008 'Novel polymer nanoparticle based drug releasing systems for improving the efficacy of drug administration in cancer chemotherapy'). Arun Kumar T. Thulasidasan acknowledges Council of Scientific and Industrial Research, New Delhi, India for providing Senior Research Fellowship. Archana P. Retnakumari acknowledges SERB-NPDF (SERB-NPDF $\mathrm{PDF} / 2016 / 000076$ ) for providing financial support.

\section{CONFLICTS OF INTEREST}

The authors disclose no potential conflicts of interest.

\section{REFERENCES}

1. Mekhail TM, Markman M. Paclitaxel in cancer therapy. Expert Opin Pharmacother. 2002; 3:755-66.

2. Jordan MA, Wilson L. Microtubules as a target for anticancer drugs. Nat Rev Cancer. 2004; 4:253-65.

3. Yared JA, Tkaczuk KH. Update on taxane development: new analogs and new formulations. Drug Des Devel Ther. 2012; 6:371-84.

4. Vasey PA. Resistance to chemotherapy in advanced ovarian cancer: mechanisms and current strategies. Br J Cancer. 2003; 89:S23-8.

5. Muñoz-Fontela C, Marcos-Villar L, Hernandez F, Gallego P, Rodriguez E, Arroyo J, Gao SJ, Avila J, Rivas C. Induction of paclitaxel resistance by the Kaposi's sarcoma-associated herpesvirus latent protein LANA2. J Virol. 2008; 82:1518-25.

6. Lagas JS, Damen CW, van Waterschoot RA, Iusuf D, Beijnen JH, Schinkel AH. P-glycoprotein, multidrugresistance associated protein 2, Сyp3a, and carboxylesterase affect the oral availability and metabolism of vinorelbine. Mol Pharmacol. 2012; 82:636-44.

7. Yusuf RZ, Duan Z, Lamendola DE, Penson RT, Seiden MV. Paclitaxel resistance: molecular mechanisms and pharmacologic manipulation. Curr Cancer Drug Targets. 2003; 3:1-19.

8. Hawkins MJ, Soon-Shiong P, Desai N. Protein nanoparticles as drug carriers in clinical medicine. Adv Drug Deliv Rev. 2008; 60:876-85.

9. Bava SV, Puliappadamba VT, Deepti A, Nair A, Karunagaran D, Anto RJ. Sensitization of taxol-induced apoptosis by curcumin involves down-regulation of nuclear factor-kappaB and the serine/threonine kinase Akt and is independent of tubulin polymerization. J Biol Chem. 2005; 280:6301-8.

10. Bava SV, Sreekanth CN, Thulasidasan AK, Anto NP, Cheriyan VT, Puliyappadamba VT, Menon SG, Ravichandran SD, Anto RJ. Akt is upstream and MAPKs are downstream of NF- $\mathrm{KB}$ in paclitaxel-induced survival signaling events, which are down-regulated by curcumin contributing to their synergism. Int J Biochem Cell Biol. 2011; 43:331-41.

11. Sreekanth CN, Bava SV, Sreekumar E, Anto RJ. Molecular evidences for the chemosensitizing efficacy of liposomal curcumin in paclitaxel chemotherapy in mouse models of cervical cancer. Oncogene. 2011; 30:3139-52.

12. Vinod BS, Antony J, Nair HH, Puliyappadamba VT, Saikia M, Narayanan SS, Bevin A, Anto RJ. Mechanistic evaluation of the signaling events regulating curcuminmediated chemosensitization of breast cancer cells to 5-fluorouracil. Cell Death Dis. 2013; 4:e505.

13. Aggarwal BB, Shishodia S, Takada Y, Banerjee S, Newman RA, Bueso-Ramos CE, Price JE. Curcumin suppresses the paclitaxel-induced nuclear factor-kappaB pathway in breast cancer cells and inhibits lung metastasis of human breast cancer in nude mice. Clin Cancer Res. 2005; 11:7490-7498.

14. Anand P, Kunnumakkara AB, Newman RA, Aggarwal BB. Bioavailability of curcumin: problems and promises. Mol Pharm. 2007; 4:807-18.

15. Liang XJ, Chen C, Zhao Y, Wang PC. Circumventing tumor resistance to chemotherapy by nanotechnology. Methods Mol Biol. 2010; 596:467-88.

16. Ganta S, Amiji M. Coadministration of Paclitaxel and curcumin in nanoemulsion formulations to overcome multidrug resistance in tumor cells. Mol Pharm. 2009; 6:928-939.

17. Nair KL, Thulasidasan AK, Deepa G, Anto RJ, Kumar GS. Purely aqueous PLGA nanoparticulate formulations of curcumin exhibit enhanced anticancer activity with 
dependence on the combination of the carrier. Int J Pharm. 2012; 425:44-52.

18. Pillai JJ, Thulasidasan AK, Anto RJ, Chithralekha DN, Narayanan A, Kumar GS. Folic acid conjugated crosslinked acrylic polymer (FA-CLAP) hydrogel for site specific delivery of hydrophobic drugs to cancer cells. J Nanobiotechnology. 2014; 12:25.

19. Pillai JJ, Thulasidasan AK, Anto RJ, Nandan C, Ashwanikumar N, Kumar GS. Curcumin entrapped folic acid conjugated PLGA-PEG nanoparticles exhibit enhanced anticancer activity by site specific delivery. RSC Adv. 2015; 5:34497.

20. Chen H, Ahn R, Van den Bossche J, Thompson DH, O'Halloran TV. Folate-mediated intracellular drug delivery increases the anticancer efficacy of nanoparticulate formulation of arsenic trioxide. Mol Cancer Ther. 2009; 8:1955-63.

21. Bravo-Cuellar A, Ortiz-Lazareno PC, Lerma-Diaz JM, Dominguez-Rodriguez JR, Jave-Suarez LF, AguilarLemarroy A, del Toro-Arreola S, de Celis-Carrillo R, Sahagun-Flores JE, de Alba-Garcia JE, Hernandez-Flores G. Sensitization of cervix cancer cells to Adriamycin by Pentoxifylline induces an increase in apoptosis and decrease senescence. Mol Cancer. 2010; 19; 9:114.

22. Bentires-Alj M, Barbu V, Fillet M, Chariot A, Relic B, Jacobs N, Gielen J, Merville MP, Bours V. NF-kappaB transcription factor induces drug resistance through MDR1 expression in cancer cells. Oncogene. 2003; 22:90-97.

23. Kennedy SG, Wagner AJ, Conzen SD, Jordán J, Bellacosa A, Tsichlis PN, Hay N. The PI3-kinase/Akt signaling pathway delivers an anti-apoptotic signal. Genes Dev. 1997; 11:701-13.

24. Edinger AL, Thompson CB. An activated mTOR mutant supports growth factor-independent, nutrient-dependent cell survival. Oncogene. 2004; 23:5654-63.

25. Chang L, Karin M. Mammalian MAP kinase signaling cascades. Nature. 2001; 410:37-40.

26. Wang CY, Cusack JC Jr, Liu R, Baldwin AS Jr. Control of inducible chemoresistance: enhanced anti-tumor therapy through increased apoptosis by inhibition of NF-kappaB. Nat Med. 1999; 5:412-7.

27. Lee MW, Kim DS, Min NY, Kim HT. Akt1 inhibition by RNA interference sensitizes human non-small cell lung cancer cells to cisplatin. Int J Cancer. 2008; 122:2380-4.

28. Woo HN, Lee WI, Kim JH, Ahn J, Han JH, Lim SY, Lee WW, Lee H. Combined antitumor gene therapy with herpes simplex virus-thymidine kinase and short hairpin RNA specific for mammalian target of rapamycin. Int $\mathrm{J}$ Oncol. 2015; 47:2233-9.

29. Gupta J, Igea A, Papaioannou M, Lopez-Casas PP, Llonch E, Hidalgo M, Gorgoulis VG, Nebreda AR. Pharmacological inhibition of p38 MAPK reduces tumor growth in patient-derived xenografts from colon tumors. Oncotarget. 2015; 6:8539-51. https://doi.org/10.18632/ oncotarget.3816.

30. Hill B. Drug resistance. Int J Oncol. 1996; 9:197-203.
31. Liu T, Li L, Fu C, Liu H, Chen D, Tang F. Pathological mechanisms of liver injury caused by continuous intraperitoneal injection of silica nanoparticles. Biomaterials. 2012; 33:2399-407.

32. Li D, Chen L, Wang H, Yang H, Dai Y, Yu C, Dong Y. Clinical application of a rapid cervical cancer screening method: Folate receptor-mediated staining of cervical neoplastic epithelia. Asia Pac J Clin Oncol. 2017; 13:44-52.

33. Mukhtar E, Adhami VM, Mukhtar H. Targeting microtubules by natural agents for cancer therapy. Mol Cancer Ther. 2014; 13:275-84.

34. Rowinsky EK, Eisenhauer EA, Chaudhry V, Arbuck SG, Donehower RC. Clinical toxicities encountered with paclitaxel (Taxol). Semin Oncol. 1993; 20:1-15.

35. Blagosklonny MV, Fojo T. Molecular effects of paclitaxel: myths and reality (a critical review). Int J Cancer. 1999; 83:151-6.

36. Aggarwal S, Ichikawa H, Takada Y, Sandur SK, Shishodia S, Aggarwal BB. Curcumin (diferuloylmethane) downregulates expression of cell proliferation and antiapoptotic and metastatic gene products through suppression of IkappaBalpha kinase and Akt activation. Mol Pharmacol. 2006; 69:195-206.

37. Aggarwal BB, Shishodia S, Takada Y, Banerjee S, Newman RA, Bueso-Ramos CE, Price JE. Curcumin suppresses the paclitaxel induced nuclear factor-kappaB pathway in breast cancer cells and inhibits lung metastasis of human breast cancer in nude mice. Clin Cancer Res. 2005; 11:7490-7498.

38. Vinod BS, Maliekal TT, Anto RJ. Phytochemicals as chemosensitizers: from molecular mechanism to clinical significance. Antioxid Redox Signal. 2013; 18:1307-48.

39. Deepa G, Thulasidasan AK, Anto RJ, Pillai JJ, Kumar GS. Cross-linked acrylic hydrogel for the controlled delivery of hydrophobic drugs in cancer therapy. Int J Nanomedicine. 2012; 7:4077-88.

40. Kapoor DN, Bhatia A, Kaur R, Sharma R, Kaur G, Dhawan S. PLGA: a unique polymer for drug delivery. Ther Deliv. 2015; 6:41-58.

41. Pavot V, Berthet M, Rességuier J, Legaz S, Handké N, Gilbert SC, Paul S, Verrier B. Poly(lactic acid) and poly(lactic-co-glycolic acid) particles as versatile carrier platforms for vaccine delivery. Nanomedicine (Lond). 2014; 9:2703-18.

42. Arunkumar R, Prashanth KV, Manabe Y, Hirata T, Sugawara T, Dharmesh SM, Baskaran V. Biodegradable Poly (Lacticco-Glycolic Acid)-Polyethylene Glycol Nanocapsules: An Efficient Carrier for Improved Solubility, Bioavailability, and Anticancer Property of Lutein. J Pharm Sci. 2015; 104:2085-93.

43. Retnakumari A, Setua S, Menon D, Ravindran P, Muhammed H, Pradeep T, Nair S, Koyakutty M. Molecularreceptor-specific, non-toxic, near-infrared-emitting $\mathrm{Au}$ cluster-protein nanoconjugates for targeted cancer imaging. Nanotechnology. 2010; 21:055103. 
44. Retnakumari A, Jayasimhan J, Chandran P, Menon D, Nair S, Mony U, Koyakutty M. CD33 monoclonal antibody conjugated Au cluster nano-bioprobe for targeted flow-cytometric detection of acute myeloid leukaemia. Nanotechnology. 2011; 22:285102.

45. Setua S, Menon D, Asok A, Nair S, Koyakutty M. Folate receptor targeted, rare-earth oxide nanocrystals for bi-modal fluorescence and magnetic imaging of cancer cells. Biomaterials. 2010; 31:714-29.

46. Sercombe L, Veerati T, Moheimani F, Wu SY, Sood AK, Hua S. Advances and Challenges of Liposome Assisted Drug Delivery. Front Pharmacol. 2015; 6:286.

47. Bae YH, Park K. Targeted drug delivery to tumors: myths, reality and possibility. J Control Release. 2011; 153:198-205.
48. Zhang HZ, Li XM, Gao FP, Liu LR, Zhou ZM, Zhang QQ. Preparation of folate-modified pullulan acetate nanoparticles for tumor-targeted drug delivery. Drug Deliv. 2010; 17:48-57.

49. Zhang L, Hou S, Mao S, Wei D, Song X, Lu Y. Uptake of folate-conjugated albumin nanoparticles to the SKOV3 cells. Int J Pharm. 2004; 287:155-62.

50. Yoo MK, Park IK, Lim HT, Lee SJ, Jiang HL, Kim YK, Choi YJ, Cho MH, Cho CS. Folate-PEG-superparamagnetic iron oxide nanoparticles for lung cancer imaging. Acta Biomater. 2012; 8:3005-13.

51. Chou TC. Drug combination studies and their synergy quantification using the Chou-Talalay method. Cancer Res. 2010; 70: 440-446. 